\title{
Os fatores de risco cardiovascular são conhecidos por estudantes do Curso de Enfermagem?
}

\author{
Are cardiovascular risk factors known to nursing students?
}

\section{¿Conocen los estudiantes de enfermería los factores de riesgo cardiovascular?}

\section{Ana Carla de Oliveira Cypriano ${ }^{1}$, Sara Cristine Marques dos Santos ${ }^{2}$, Eduarda de Oliveira Chagas ${ }^{3}$, Thaís Lemos de Souza Macedo ${ }^{4}$, Ivan Lucas Picone Borges dos Anjos 5 , Ivana Picone Borges de Aragão6}

Como citar esse artigo. Cypriano, A.O.C dos Santos, S.C.M; Chagas, E.O; Macedo, T.L.S; dos Anjos, I.L.P.B; de Aragão, I.P.B. Os fatores de risco cardiovascula são conhecidos por estudantes do Curso de Enfermagem? Revista Pró-UniverSUS. 2021 Jul./Dez.; 12 (2): 02-06

\section{Resumo}

Mundialmente, a ocorrência de doença cardiovascular no sexo feminino está em ascensão, observa-se que há sub-diagnóstico e sub-tratamento, aumentando os riscos de desfechos não favoráveis, como incapacidade e morte. Os sintomas em mulheres podem ser atípicos e não alertarem para a saúde cardiovascular, causando um atraso maior na busca de orientação médica. Estudo observacional, transversal, quantitativo e qualitativo com a participação de 31 alunas do curso de enfermagem durante o período de junho a novembro de 2020 e através da coleta de respostas de um questionário anônimo. Foi obtido que apesar de cerca de $70 \%$ das alunas apresentarem sintomas compatíveis com afecções cardiovasculares, apenas $6 \%$ procuraram atendimento cardiológico para uma investigação mais minuciosa. O estudo evidenciou a necessidade de maior conscientização da mulher no cuidado com a saúde, sobretudo do coração.

Palavras-chave: Prevenção Primária; Fatores de Risco Cardiovascular; Doenças Cardiovasculares.

\begin{abstract}
Worldwide, the occurrence of cardiovascular disease in women is on the rise, it is observed that there is underdiagnosis and under-treatment, increasing the risks of non-favourable outcomes, such as disability and death. Symptoms in women may be atypical and not alert to cardiovascular health, causing a greater delay in seeking medical advice. Observational, cross-sectional, quantitative and qualitative study with the participation of 31 students of the nursing course during the period from June to November 2020 and through the collection of answers from an anonymous questionnaire. It was obtained that although about $70 \%$ of the students presented symptoms compatible with cardiovascular disorders, only $6 \%$ sought cardiological care for a more thorough investigation. The study showed the need for greater awareness of women in health care, especially the heart. Keywords: Primary Prevention. Cardiovascular risk factors. Cardiovascular Diseases.
\end{abstract}

\section{Resumen}

En todo el mundo, la incidencia de las enfermedades cardiovasculares en las mujeres va en aumento, se observa que hay un infradiagnóstico y un infratratamiento, lo que aumenta los riesgos de resultados no favorables, como la discapacidad y la muerte. Los síntomas en las mujeres pueden ser atípicos y no alertar sobre la salud cardiovascular, lo que provoca un mayor retraso en la búsqueda de asesoramiento médico. Estudio observacional, transversal, cuantitativo y cualitativo con la participación de 31 alumnos del curso de enfermería durante el periodo de junio a noviembre de 2020 y mediante la recogida de respuestas de un cuestionario anónimo. Se obtuvo que, aunque cerca del 70\% de los estudiantes presentaban síntomas compatibles con trastornos cardiovasculares, sólo el 6\% buscó atención cardiológica para una investigación más exhaustiva. El estudio puso de manifiesto la necesidad de una mayor concienciación de las mujeres en el ámbito de la salud, especialmente del corazón.

Palabras clave: Prevención primaria. Factores de riesgo cardiovascular. Enfermedades cardiovasculares.

Afiliação dos autores:

Discente do curso de enfermagem da Universidade de Vassouras, Vassouras, RJ, Brasil. ORCID: https://orcid.org/0000-0003-2517-0607

${ }_{2}^{2}$ Discente do curso de Medicina da Universidade de Vassouras, Vassouras, RJ, Brasil. ORCID: https://orcid.org/0000-0002-8205-8112

${ }^{3}$ Discente do curso de enfermagem da Universidade de Vassouras, Vassouras, RJ, Brasil. ORCID: https://orcid.org/0000-0002-7621-3710

${ }^{4}$ Discente do curso de Medicina da Universidade de Vassouras, Vassouras, RJ, Brasil. ORCID: https://orcid.org/0000-0002-7667-6061

${ }^{5}$ Discente do curso de Medicina da Universidade de Vassouras, Vassouras, RJ, Brasil. ORCID: https://orcid.org/0000-0003-4211-1887

${ }^{6}$ Professora do curso de Medicina da Universidade de Vassouras, Vassouras, RJ, Brasil. ORCID: https://orcid.org/0000-0002-4295-0165 


\section{Introdução}

A incidência de doença cardiovascular (DCV) na mulher vem aumentando no mundo. Os sintomas de doença arterial coronária na angina ou no infarto agudo do miocárdio (IAM) podem cursar de forma diferentes nesse grupo, levando a uma demora na sua detecção e falha no tratamento ${ }^{1}$. A DCV feminina tende a cursar com o acometimento de vasos menores e por isso ocorre a não equivalência dos sintomas entre os dois $\operatorname{sexos}^{2}$. Acredita-se que a exclusão das mulheres de estudos clínicos na área de cardiologia nas décadas anteriores, somada a crença de que elas estão mais predispostas ao câncer em detrimento as DCV seja uma das causas do crescimento da mortalidade cardiovascular feminina ${ }^{3}$. A DCV em mulheres é notadamente subdiagnosticada, especialmente, nas mais jovens, sendo detectada geralmente após um segundo episódio de infarto, causando maiores repercussões debilitantes a sua saúde ${ }^{4}$.

Os fatores de risco cardiovascular (FRC) em mulheres, quando comparados aos do sexo masculino, apresentam perfil mais deletério, sendo agravado pelo fato de serem subestimados, devido aos escores de risco tradicionais, como Framingham, não considerarem as especificidades hormonais femininas. Essas particularidades, englobam além dos FRC tradicionais, a exposição aos FR hormonais e as situações de risco como a síndrome dos ovários policísticos $(\mathrm{SOP})^{5}$. O FR hormonal é especifico e tem três fases principais, a contracepção, gravidez e menopausa onde existe a associação do risco cardiovascular com o metabólico e hábitos saudáveis de vida podem prevenir ou abrandar a progressão e mortalidade cardiovascular feminina causada por essa transição metabólico-vascular ${ }^{5}$. A menarca tardia ou precoce se correlaciona, fortemente, com a incidência de doenças cardiovasculares e, de forma mais sutil, com a doença cerebrovascular e hipertensão ${ }^{6}$.

As mulheres jovens contam com maior exposição aos fatores psicossociais como a ansiedade, depressão, estresse e as condições socioeconômicas e esses fatores podem estar associados a um mau prognóstico cardiovascular5. A hipertensão arterial e diabetes mellitus aumentam o risco de IAM e, o exercício físico, é mais cardioprotetor em mulheres, quando comparados aos homens ${ }^{7}$. Sendo que, comparativamente, os homens praticam mais atividade física que as mulheres, que passam muito tempo sentada e, quando decidem iniciar processo de dieta, o exercício físico é o menos prevalente na rotina ${ }^{5,8}$.

$\mathrm{O}$ risco de mortalidade cardiovascular é $6 \%$ maior para mulheres do que comparadas aos homens 9 . O impacto dos FRC tradicionais na vida varia de acordo com o sexo e, a prevalência, aumenta de acordo com a idade, nas mulheres são observados mais FRC que nos homens de idade semelhante, explicado estilo de vida, somado ao estresse psicossocial ${ }^{5}$. O estresse tem ação no sistema nervoso e, juntamente, à elevação dos triglicérides, com maior predominância em mulheres, podem agravar quadros ateroscleróticos e endoteliais ${ }^{4,10}$. Comparativamente, aos homens, as mulheres são mais propensas ao estresse devido a sua rotina multitarefas, sendo o IAM e o acidente vascular cerebral (AVC), a maior causa de óbitos entre elas, além de superior ao câncer de mama. As DCV são 49\% mais incidentes nos óbitos que as neoplasias mamárias ${ }^{10,11}$.

Apesar dos avanços nas pesquisas e das campanhas de conscientização da incidência de DCV em mulheres, elas são menos propensas a receberem recomendações mais focadas das diretrizes de diagnóstico e tratamento. A maior preocupação feminina e dos profissionais que realizam o atendimento primário, é o câncer de mama, onde é direcionado o foco para esse rastreamento e em detrimento às $\mathrm{DCV}$, que irão se desenvolver lentamente, partindo do princípio que $90 \%$ das mulheres possuem ao menos $1 \mathrm{FRCV}$ e, para a prevenção mais eficaz, é necessário um tratamento precoce ${ }^{9}$, portanto, é mandatório o rastreamento precoce.

O objetivo do presente estudo foi fazer um levantamento do conhecimento dos fatores de risco cardiovascular por mulheres do curso de enfermagem de uma universidade privada.

\section{Metodologia}

Estudo observacional, transversal, quantitativo e qualitativo com a participação de 31 alunas do curso de enfermagem. Ocorrido durante o período de junho a novembro de 2020. O instrumento da coleta de dados foi um questionário online anônimo relacionado a pesquisa de FCR e autopercepção de estresse com parecer do Conselho de Ética em Pesquisa (CEP) de número 4.166.747.

Foram avaliados conhecimento acerca de níveis de pressão arterial (PA), uso de medicação para controle da PA, conhecimento dos valores de colesterol, índice de massa corporal (IMC), histórico familiar para DCV, sintomas cardiovasculares, gravidez, intercorrências durante a gestação e frequência no rastreamento ginecológico e cardiovascular, queixa de sintomas compatíveis com os presentes nas cardiovasculares. Foram questionadas sobre sete sintomas cardiovasculares para que as participantes pudessem assinalar os que estivessem presentes no seu dia a dia. As limitações da pesquisa foram a baixa adesão das alunas e a impossibilidade de um acompanhamento sintomatológico por um médico, devido ao anonimato da pesquisa. 


\section{Resultados}

Total de 31 mulheres participaram do estudo, com média de idade de 22 anos; máximo de 53 e mínimo de 18 anos.

O tabagismo foi negado por 28(90,32\%); positivo em $1(3,23 \%)$ e $2(6,45 \%)$ ex-fumantes. A PA $>120 \times 80 \mathrm{mmHg}$ foi negada por $26(83,87 \%)$; afirmada por $3(9,68 \%)$ e $2(6,45 \%)$ não sabem. $O$ uso de antihipertensivo está presente em 3(9,68\%). A média de PAS informada foi de $112 \mathrm{mmHg}$; mediana de 110; desvio padrão de 23,50; com valor máximo de $220 \mathrm{mmHg}$, mínimo de $90 \mathrm{mmHg}$ e nível de confiança (95\%) foi de 9,11, onde entre aquelas que informaram, sendo $28(90,32 \%)$ ). Quanto a PAD, 3(9,68\%) desconhecem e $28(90,32 \%$, ) informaram, onde obteve-se que a média é de $74 \mathrm{mmHg}$; mediana de 80 ; desvio padrão de 11,37; valor máximo $110 \mathrm{mmHg}$, mínimo $60 \mathrm{mmHg}$ e nível de confiança $(95 \%)$ de 4,4 .

Do total, 22(70,97\%) afirmaram já terem realizado exame de colesterol; $6(19,35 \%)$ não e $3(9,68 \%)$ não sabem. A minoria, 3(9,68\%), possuem colesterol acima de $190 \mathrm{mg} / \mathrm{dl} ; 11(35,48 \%)$ negam, porém, $17(54,84 \%)$ desconhecem. Somente 5(16,13\%) soube informar o valor do colesterol, com média de $166 \mathrm{mg} / \mathrm{dl}$; desvio padrão de 23,02; valor máximo de $200 \mathrm{mg} / \mathrm{dl}$, mínimo de $140 \mathrm{mg} / \mathrm{dl}$ e nível de confiança $(95 \%)$ de 28,58. Em relação à fração HDL, 3(9,68\%) relataram que era $<40 \mathrm{mg} / \mathrm{dl} ; 5(16,13 \%)$ negam e $23(74,19 \%)$ não sabem. Apenas 1(3,23\%) informou o valor, sendo esse de valor $47 \mathrm{mg} / \mathrm{dl}$. Nenhuma participante referiu uso de medicamento para controle do colesterol.

Todas as mulheres avaliadas negaram IAM ou angina entre seus antecedentes pessoais. No histórico familiar, $5(16,13 \%)$ relatam que pai ou irmão tiveram infarto, AVE ou problema cardíaco antes dos 55 anos; já quanto a mãe ou irmã, $4(12,9 \%)$.

$\mathrm{O}$ exame de glicemia refere ter sido realizado por $24(77,42 \%), 2(6,45 \%)$ não fizeram e $5(16,13 \%)$ não sabem. Ser portadora de diabetes mellitus e/ou taxa de glicose superior a $126 \mathrm{mg} / \mathrm{dl}$ foi relatada por $2(6,45 \%)$, $23(74,19 \%)$ negam e 6(19,35\%) desconhecem. A medicação para controle glicêmico é realizada por $3(9,68 \%)$.

O peso foi informado por $30(96,77 \%)$ participantes, onde a média de peso foi $64 \mathrm{~kg}$; desvio padrão de 10,10; o valor máximo foi $91 \mathrm{~kg}$, mínimo $50 \mathrm{~kg}$ e o nível de confiança (95\%) de 3,77.

A altura foi informada por todas as participantes, com média de $164 \mathrm{~cm}$; desvio padrão de 5,6; valor máximo de $176 \mathrm{~cm}$, mínimo de $150 \mathrm{~cm} 50 \mathrm{~kg}$ e o nível de confiança $(95 \%)$ de 2,05 .

O IMC > 25 foi relatado por 7(22,58\%); negado por $16(51,61 \%)$ e $8(25,81 \%)$ desconhecem. A maioria das participantes, 20(64,5\%), informou o valor do IMC, havendo desconhecimento em 11(35,5\%). A média foi de 23,34; mediana de 22,25; desvio padrão de 3,67; com máximo de 30,1, mínimo de 15 e o nível de confiança (95\%) foi de 1,72 .

O exercício físico por mais de 30 minutos na maioria dos dias da semana, totalizando 150 minutos por semana, foi relatado por $11(35,48 \%)$; negado por $19(61,29 \%)$ e 1(3,23\%) não sabem.

A fadiga foi relatada por $25(80,65 \%)$; palpitação por $16(51,61 \%)$; falta de ar por $21(67,74 \%)$; desmaio sem explicação por $1(3,23 \%)$; dor nas pernas ao caminhar por $11(35,48 \%)$; desconforto ou dor no peito aos esforços por $8(25,81 \%)$ e ao repouso por $7(22,58 \%)$.

A soma dos sintomas mostrou que $22(70,97 \%)$ possuem 2 ou mais sintomas e $9(29,03 \%) 1$ ou nenhum. De forma mais detalhada, $9(10,11 \%)$ referiram 1 sintoma; $10(11,24 \%)$ com 2 ; $9(10,11 \%)$ com $3 ; 36(40,45 \%)$ com 4 e $25(28,09 \%)$ com 5 .

O uso de anticoncepcional foi referido por $15(48,39 \%)$, uso prévio em $4(12,9 \%)$ e $12(38,71 \%)$ nunca usaram. Todas negaram gravidez no momento da pesquisa e $27(87,1 \%)$ nunca engravidaram;

A menopausa foi relatada por $1(3,23 \%)$ pelo período pregresso de 3 anos e negada pelas demais. A maioria, 29(93,55\%), negou passado de histerectomia e, $2(6,45 \%)$, desconheciam. A ooforectomia unilateral foi relatada por $1(3,23 \%)$ há 19 anos.

Avaliação médica ginecológica foi relatada por $16(51,61 \%)$ e negado por $15(48,39 \%)$, porém, avaliação cardiológica foi identificada apenas em $2(6,45 \%)$ e $29(93,55 \%)$ negam.

Aautopercepção do estresse foi de que 21(67,74\%) afirmaram ser muito frequente; $6(19,35 \%)$ às vezes; $1(3,23 \%)$ pouco frequente e $3(9,68 \%)$ nunca (figura 1$)$.

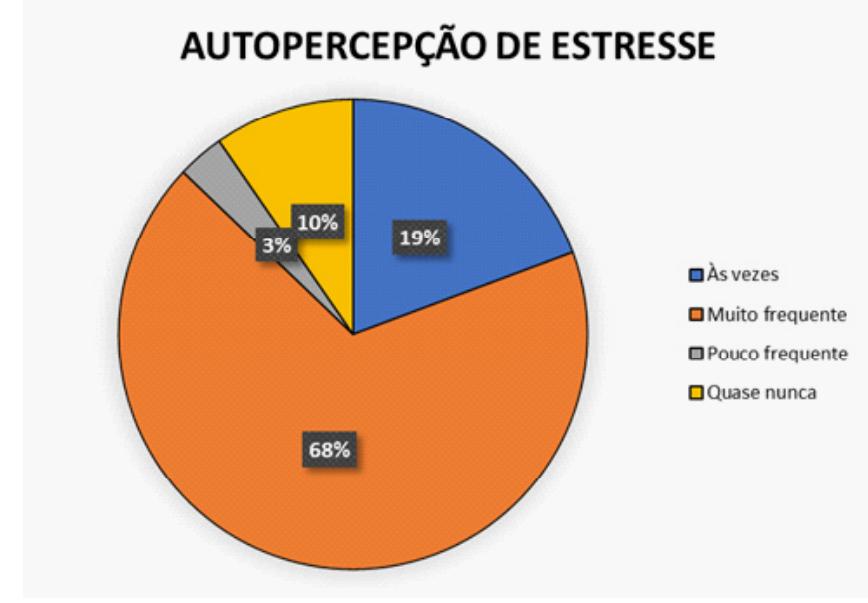

Figura 1. gráfico de autopercepção do estresse entre as acadêmicas de enfermagem. 


\section{Discussão}

$\mathrm{O}$ presente estudo demonstrou haver desconhecimento em relação aos FRC associado à informação de sintomatologia compatível e falta de rastreamento de DCV nas estudantes de enfermagem avaliadas. O aumento da incidência de internações de mulheres jovens por DCV é uma realidade preocupante, visto que pode resultar em incapacidade ou morte ${ }^{12,13}$. A identificação dos FRC constitui uma ferramenta de importância na tentativa de redução do impacto da DCV, sendo necessário identificar as mulheres portadoras e atuar naqueles que são modificáveis ${ }^{12}$.

A maioria das mulheres participantes desse estudo negou tabagismo, considerado um dos maiores preditores de risco para DAC, sobretudo o IAM ${ }^{14}$.

A hipertensão é um importante FRC, sendo multifatorial, onde interações ambientais e genéticas podem alterar os níveis pressóricos ${ }^{15}$. Nesse estudo, os valores de PA superiores a $120 \times 80 \mathrm{mmHg}$ foi negado por $83 \%$ das entrevistadas, independentemente, de haver uso de medicação anti-hipertensiva, por $9 \%$. Demonstrando pouco desconhecimento, $2(6,45 \%)$, acerca dos próprios níveis tencionais arteriais.

Do total de estudantes, $70 \%$ já realizaram a medida do colesterol, porém mais da metade desconhece o valor e apenas 5 souberam informar. Cerca de $3 / 4$ desconheciam, também, o valor de HDL. Sabe-se que as elevações dos níveis lipídicos séricos constituem FRC e as causas estão relacionadas ao estilo e vida ${ }^{16}$. Sua medição é importante, assim como o conhecimento a respeito dos valores para acompanhamento.

$\mathrm{O}$ exame de glicemia foi realizado pela maioria do grupo, sendo negado a presença de diabetes mellitus e/ou taxa de glicose superior na maioria. $\mathrm{O}$ desconhecimento foi evidenciado na minoria, o que vem de encontro com a importância da prevenção primária e conscientização dos fatores de risco cardiovasculares na população. Estima-se que a hiperglicemia pode lesar o endotélio pela glicosilação de LDLs, iniciando um processo inflamatório que pode progredir para a aterosclerose, ou seja, o aumento da glicemia se relacionada continuamente com o desenvolvimento de DCV ou pela elevada resposta insulinêmica, que predispõe dislipidemia, hipertensão arterial e disfunção do endotélio ${ }^{17}$.

A maioria do grupo conhecia seu IMC, sendo demonstrado que os valores médios eram compatíveis com normalidade, apesar da baixa adesão à prática de exercício físico. Considerada como fator de risco independente, a obesidade é um problema de saúde em ascensão, relacionando-se com o desenvolvimento de doenças crônicas $^{18}$, reduzindo a expectativa de vida e sendo causa de mortalidade principalmente em mulheres ${ }^{19}$. O IMC é utilizado para mensurar o excesso de peso/estado nutricional, estando associado aos demais fatores de risco cardiovascular ${ }^{18,19}$. Já a prática de atividades físicas é considerada um estimulante da função cardiovascular, atuando como prevenção primária de doenças crônicas ${ }^{20}$.

Quanto a sintomatologia, a fadiga foi a mais prevalente, relatada por $80 \%$ das mulheres, seguida de falta de ar e palpitação. Devem ser questionados aqui a possibilidade de existir a DCV ou mesmo a associação ao estresse, a rotina de multitarefas e ao cansaço. Entre os sinais de exaustão e estresse, pode-se mencionar sudorese aumentada, hipertensão arterial, taquicardia, náuseas, tensão muscular e etc ${ }^{21}$. Desmaio sem explicação, dor nas pernas ao caminhar e dor no peito mediante ou não exercício físico foi relatado por menos mulheres. Ao final, $70 \%$ das entrevistadas possuíam 2 ou mais sintomas.

Os sintomas avaliados são comuns aos identificados nas doenças cardiovasculares, não sendo observado, no presente estudo, aumento da procura por avaliação cardiológica. A avaliação médica ginecológica foi relatada em mais de $50 \%$ do grupo, apesar da baixa evidência de informação sobre a avaliação cardiológica em apenas em 2(6,45\%). É observado que a mulher não tem por hábito investigar sintomas de origem cardiovascular, negligenciando a ida ao cardiologista mesmo na ocorrência de sintomas ${ }^{22}$. Ainda, é importante ressaltar a atipia sintomática ocorrida na mulher mediante um IAM, fazendo com que haja um subdiagnóstico de casos mais graves e atraso no atendimento hospitalar ${ }^{23}$.

$\mathrm{O}$ presente estudo evidenciou a necessidade de progressiva conscientização acerca dos FRC em mulheres, que contribuirão para a redução da DCV em mulheres, que constituem a maior causa de óbito em mulheres no mundo, segundo a Organização Mundial de Saúde ${ }^{24-26}$.

\section{Conclusão}

O presente evidenciou que a maioria das mulheres estudantes de enfermagem demonstravam peso corporal normal, não eram aderentes ao tabagismo, conheciam que os valores da PA eram abaixo de ideais, além de já terem sido submetidas ao exame laboratorial de glicemia e colesterolemia. Porém, a maioria desconhecia os valores de colesterolemia.

Sintomas comuns ao sistema cardiovascular foram relatados em mais da metade das mulheres, como palpitações ou falta de ar, associado à prática de exercício físico de 150 minutos por semana em menos da metade do grupo. Apesar da sintomatologia, o estudo demonstrou que a avaliação cardiológica ocorreu em menos de $10 \%$ do grupo. 


\section{Referências}

1. Women and Heart Disease | CardioSmart - American College of Cardiology [Internet]. [citado 9 de dezembro de 2020]. Available at: https:// www.cardiosmart.org/topics/women-and-heart-disease

2. Women and heart disease $\mid$ Heart and Stroke Foundation [Internet]. [citado 26 de dezembro de 2020]. Available at: https://www.heartandstroke. $\mathrm{ca} /$ heart-disease/what-is-heart-disease/types-of-heart-disease/women-andheart-disease

3. Figueiredo JHC. Estresse, Mulheres e Infarto Agudo do Miocárdio: O que se Sabe? Arq Bras Cardiol [Internet]. 13 de outubro de 2020 [citado 15 de dezembro de 2020];115(4):658-9. Available at: http://abccardiol.org/ en/short-editorial/stress-women-and-acute-myocardial-infarction-what-isknown/

4. WHO | The Atlas of Heart Disease and Stroke. WHO. 2010;

5. Mounier-Vehier C, Nasserdine P, Madika A-L. [Stratification of cardiovascular risk in women: Optimize the medical care]. Presse Med. novembro de 2019;48(11 Pt 1):1249-56.

6. Dexter C, Valerie B, Angela B, Lucy WF, E. KM, K. RG, et al. Age at Menarche and Risks of Coronary Heart and Other Vascular Diseases in a Large UK Cohort. Circulation [Internet]. 20 de janeiro de 2015;131(3):23744. Available at: https://doi.org/10.1161/CIRCULATIONAHA.114.010070

7. Yusuf S, Hawken S, Ounpuu S, Dans T, Avezum A, Lanas F, et al. Effect of potentially modifiable risk factors associated with myocardial infarction in 52 countries (the INTERHEART study): case-control study. Lancet (London, England). setembro de 2004;364(9438):937-52.

8. Kotseva K, De Bacquer D, De Backer G, Rydén L, Jennings C, Gyberg V, et al. Lifestyle and risk factor management in people at high risk of cardiovascular disease. A report from the European Society of Cardiology European Action on Secondary and Primary Prevention by Intervention to Reduce Events (EUROASPIRE) IV cross-sectional su. Eur J Prev Cardiol [Internet]. 27 de dezembro de 2016;23(18):2007-18. Available at: http:// journals.sagepub.com/doi/10.1177/2047487316667784

9. L. BH, J. WJ, Eugenia G, Martha G, J. HA, M. HL, et al. Promoting Risk Identification and Reduction of Cardiovascular Disease in Women Through Collaboration With Obstetricians and Gynecologists: A Presidential Advisory From the American Heart Association and the American College of Obstetricians and Gynecologi. Circulation [Internet]. 12 de junho de 2018;137(24):e843-52. Available at: https://doi.org/10.1161/ CIR.0000000000000582

10. Schmidt K, Lima A da S, Schmitt KR, Moraes MA, Schmidt MM. Um Olhar sobre o Stress nas Mulheres com Infarto Agudo do Miocárdio. Arq Bras Cardiol [Internet]. 13 de outubro de 2020 [citado 15 de dezembro de 2020];115(4):649-57. Available at: http://abccardiol.org/en/article/stressin-women-with-acute-myocardial-infarction-a-closer-look/

11. Fernandes CE, Pinho-Neto JSL, Gebara OCE, Santos Filho RD, Pinto Neto AM PFA, Athayde AVL, Sposito AC, Ferrari AEM, Albergaria BH, Silva CR, Arruda CG, Stephan C, Nahas EP, Pellini EAJ, Alexandre ERG CE, Porto E, Lima GR, Andrade IALB, Ferreira JAS, Lima JC, Aldrighi JM, Machado LV, Azevedo LH, Pompei LM, Bertolami M, Steiner ML A, MA, Sá MFS, Wender COM, Melo NR, Spritzer PM, Strufaldi R, Machado RB, Bossemeyer RP, Costa RR, Peixoto S CV. I Diretriz Brasileira sobre Prevenção de Doenças Cardiovasculares em Mulheres Climatéricas e a Influência da Terapia de Reposição Hormonal (TRH) da Sociedade Brasileira de Cardiologia (SBC) e da Associação Brasileira do Climatério (SOBRAC). Arq Bras Cardiol. 2008;91:1-23.

12. Bonotto GM, Mendoza-Sassi RA, Susin LRO. Conhecimento dos fatores de risco modificáveis para doença cardiovascular entre mulheres e seus fatores associados: um estudo de base populacional. Cien Saude Colet [Internet]. janeiro de 2016;21(1):293-302. Available at: http://www.scielo.br/scielo.php?script=sci_arttext\&pid=S1413$81232016000100293 \& \operatorname{lng}=\mathrm{pt} \& \operatorname{tlng}=\mathrm{pt}$

13. Pagan Lassalle P, DeBlois JP, Keller A, Stoner L, Heffernan KS. Central Blood Pressure and Subclinical Atherosclerotic Risk in Young Hispanic American Women. Ethn Dis [Internet]. 21 de outubro de 2021;31(4):489-500. Available at: https://www.ethndis.org/edonline/index. $\mathrm{php} / \mathrm{ethndis/article/view/1590}$
14. Silva MAM da, Rivera IR, Ferraz MRMT, Pinheiro AJT, Alves SW dos S, Moura AÁ, et al. Prevalência de fatores de risco cardiovascular em crianças e adolescentes da rede de ensino da cidade de Maceió. Arq Bras Cardiol. 2005;84:387-92.

15. Inácio TB, Machado MC. Hipertensão e fatores de risco cardiovascular de um grupo de indivíduos adultos atendidos no ambulatório de especialidades médicas da UNISUL. Arq Bras Cardiol. 2005;85(1).

16. Salvaro RP, Ávila Junior S. Perfil lipídico e sua relação com fatores de risco cardiovascular em estudantes de nutrição. Rev SOCERJ. 2009;22(5):309-17.

17. Carvalho GQ, Alfenas R de CG. Índice glicêmico: uma abordagem crítica acerca de sua utilização na prevenção e no tratamento de fatores de risco cardiovasculares. Rev Nutr [Internet]. outubro de 2008;21(5):577-87. Available at: http://www.scielo.br/scielo.php?script=sci_arttext\&pid=S1415$52732008000500010 \& \operatorname{lng}=\mathrm{pt} \& \operatorname{lng}=\mathrm{pt}$

18. Carlucchi EM de S, Gouvêa JAG, Oliveira AP de, Silva JD da, Cassiano ACM, Bennemann RM. Obesidade e sedentarismo: fatores de risco para doença cardiovascular. Comun ciênc saúde. 2013;375-84.

19. Gus M, Moreira LB, Pimentel M, Gleisener ALM, Moraes RS, Fuchs FD. Associação entre diferentes indicadores de obesidade e prevalência de hipertensão arterial. Arq Bras Cardiol [Internet]. fevereiro de 1998;70(2):111-4. Available at: http://www.scielo.br/scielo.php?script=sci arttext\&pid=S0066-782X1998000200008\&lng=pt\&nrm=iso\&tlng=pt

20. Olbrich SR, Nitsche MJT, Neto JO, Mori NLR. Sedentarismo: prevalência e associação de fatores de risco cardiovascular. Rev Ciência em Extensão. 2009;5(2):30-41.

21. Prosdocimo ACMG, Lucina LB, Kato M da PAC de A, Macedo RM de, Cilião MR, Costantini CO, et al. Sintomas de estresse identificados pelos participantes de um programa de prevenção de doenças cardiovasculares. Rev Soc Cardiol Estado Säo Paulo. 2012;22-6.

22. Yu J, Park K, Chandrasekhar J, Kalkman DN, Johnson JA, Wild RA, et al. Feasibility and Utility of a Cardiovascular Risk Screening Tool in Women Undergoing Routine Gynecology Evaluation. J Women's Heal [Internet]. 13 de julho de 2020;29(9):1150-9. Available at: https://doi. org/10.1089/jwh.2019.8074

23. de Araújo Gouveia V. Atitudes pré-hospitalares frente aos sintomas sugestivos de IAM, em pacientes atendidos em uma emergência cardiológica do Recife. Universidade Federal de Pernambuco; 2009.

24. World Health Organization. The top 10 causes of death [Internet]. Available at: https:/www.who.int/en/news-room/fact-sheets/detail/the-top10-causes-of-death

25. Heart disease is the leading cause of death in women - here's how we can address it - Her Heart [Internet]. [citado 2 de novembro de 2021]. Available at: https://herheart.org/heart-disease-is-the-leading-cause-ofdeath-in-women-heres-how-we-can-address-it/

6. Leading Causes of Death-All races and origins-Females - United States, 2017 | Women's Health | CDC [Internet]. [citado 2 de novembro de 2021]. Available at: https://www.cdc.gov/women/lcod/2017/all-racesorigins/index.htm 